\title{
OH maser observations using the Russian interferometric network "Quasar" in preparation for scientific observations with the space mission RadioAstron.
}

\author{
I. D. Litovchenko ${ }^{1}$, A. V. Alakoz ${ }^{1}$, V. I. Kostenko ${ }^{1}$, S. F. Lihachev ${ }^{1}$, \\ A. M. Finkelstein ${ }^{2}$ and A. V. Ipatov ${ }^{2}$ \\ ${ }^{1}$ Astro Space Center of the Lebedev Physical Institute, Moscow, Russia \\ email: grosh@asc.rssi.ru \\ ${ }^{2}$ Institute of Applied Astronomy of RAS, Saint Petersburg, Russia
}

\begin{abstract}
We present results of a VLBI experiment at a wavelength of $18 \mathrm{~cm}$, which simulates the ground-space interferometer with space link to RadioAstron. An array of five antennas was used, four of them are located in the Russian Federation, plus the the 32-m radio telescope in Medicine (Italy). The 22-m radio telescope in Pushchino (Moscow Region) acted in place of the space arm. It has an effective area of 100 square meters. The three other Russian 32-m antennas are operated by the Institute of Applied Astronomy RAS; they are located at Badary, Svetloe and Zelenchukskaya (interferometer network "Quasar"). The maximum base-line, Badary-Svetloe, was about $4402 \mathrm{~km}$, providing an angular resolution of about 0.009 arc seconds at a wavelength of $18 \mathrm{~cm}$. The duration of the experiment was 10 hours on 02/03 February 2011. The program of observations included quasars $3 \mathrm{C} 273,3 \mathrm{C} 279,3 \mathrm{C} 286$ and the maser source - W3(OH). W3 $(\mathrm{OH})$ was observed only by the Russian telescopes and was investigated at the frequency of the 1665 $\mathrm{MHz}$ main line. The data were recorded on the MK5 recorder (32-m radio telescopes) and the RDR system (RadioAstron Digital Recorder) in Pushchino. The low SEFD (system equivalence of flux density) of Pushchino emulated the RadioAstron antenna. Correlation was performed with the universal software correlator of the AstroSpace Center of Lebedev Physical Institute. The correlator output format is compatible with that used by the AIPS package, which was used for data analysis. After analyzing the correlated data we obtained relative coordinates of the maser components. The main results are tabulated and presented in the figures. The data quality is sufficient for astrophysical analysis and comparison with previous observations of maser source W3(OH) on VLBI networks EVN and VLBA.
\end{abstract}

Keywords. interferometry, masers, hydroxyl, W3(OH)

\section{Radio telescopes that observed the maser radio source $\mathrm{W} 3(\mathrm{OH})$}

Three 32-m fully steerable reflectors of the Institute of Applied Astronomy RAS were used and we list their locations and other parameters below.

1. Svetloe, $\phi=60^{\circ} 32^{\prime}, \lambda=29^{\circ} 47^{\prime}, h=86 \mathrm{~m}$.

2. Zelenchukskaya, $\phi=43^{\circ} 47^{\prime}, \lambda=41^{\circ} 34^{\prime}, h=1175 \mathrm{~m}$.

3. Badary, $\phi=51^{\circ} 46^{\prime}, \lambda=102^{\circ} 14^{\prime}, h=813 m$.

All these antennas have five dual circular polarization receivers for the wavelengths: 1.35-cm (K-band), 3.5-cm (X-band), 6.2-cm (C-band), 13-cm (S-band) and 18-21-cm (L-band). Mark5B VLBI recorders were used. The SEFD at L-band is about $280 \mathrm{Jy}$.

4. The fourth antenna was the 22 -m reflector in Pushchino, $\phi=54^{\circ} 49^{\prime}, \lambda=37^{\circ} 38^{\prime}$, $h=239 m$. 


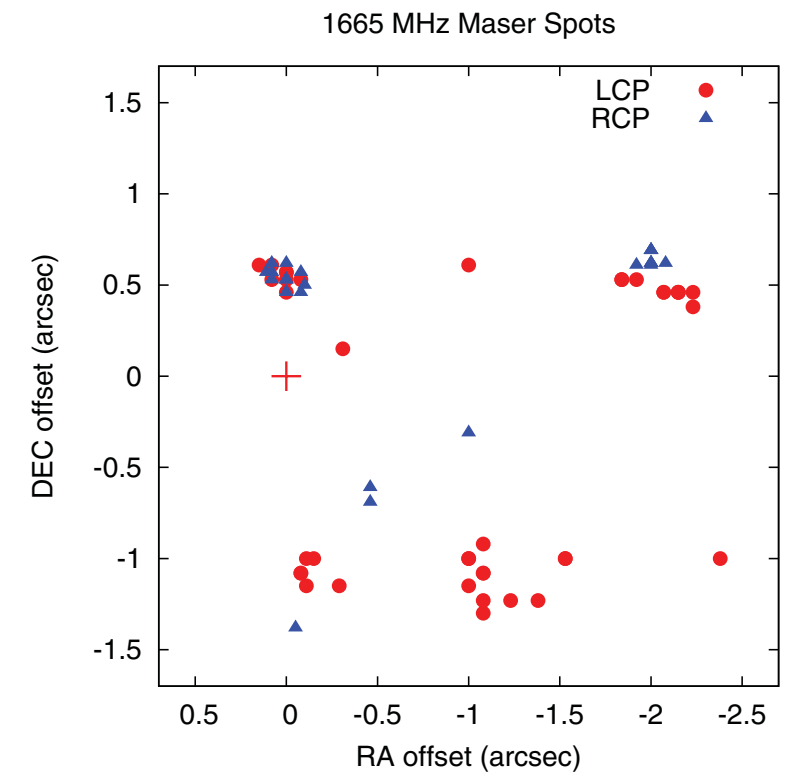

Figure 1. The 1665-MHz maser spot map of $\mathrm{W} 3(\mathrm{OH})$. Dots indicate emission in LCP and triangels indicate emission in RCP. The cross marks the phase centre with coordinates: RA $02^{h} 27^{m} 03^{s} .825$ and DEC $61^{\circ} 52^{\prime} 2^{\prime \prime} .653$.

It has receivers for 1.35-cm (K-band), 6.2-cm (C-band), 18-21-cm (L-band) and 92-cm (P-band).

In this experiment we used RadioAstron recording system RDR (RadioAstron Digital Recorder). The SEFD at L-band is about $1000 \mathrm{Jy}$.

\section{Correlation}

Correlation was performed with the universal software correlator of the AstroSpace Center of Lebedev Physical Institute. The correlator output format is compatible with that used by the AIPS package. The final data had 6148 frequncy channels, bandwidth $3.002 \mathrm{MHz}$ with channel separation $0.4883 \mathrm{kHz}$.

\section{Data analysis}

To obtain the positions of maser spots of $\mathrm{W} 3(\mathrm{OH})$ we used the AIPS fringe-rate mapping task FRMAP. Then we determined the positions of the masers from lines of intersection on the fringe-rate maps. Results for all channels and both polarizations are shown on Fig. 1 which shows the relative postions of individual maser features. These are well matched to the map obtained by Wright et al. 2004 .

\section{References}

Wright, M. M., Gray, M. D., \& Diamond P. J. 2004, MNRAS, 350, 1253 